\title{
Problem with the superstring action of Deriglazov and Galajinsky
}

\author{
Nathan Berkovits* \\ Instituto de Física Teórica, Universidade Estadual Paulista, Rua Pamplona 145, São Paulo, SP 01405-900, Brazil \\ (Received 12 January 1998; published 28 January 1999)
}

\begin{abstract}
Deriglazov and Galajinsky have recently proposed a new covariant action for the Green-Schwarz superstring which can be constructed in any spacetime dimension. In this Comment, I show that their action contains extra on-shell degrees of freedom as compared with the standard action and is therefore inequivalent. [S0556-2821(99)00704-3]
\end{abstract}

PACS number(s): 11.25.Hf, 11.30.Pb

In five recent papers $[1,2,3,4,5],{ }^{1}$ Deriglazov and Galajinsky have proposed a new covariant action for the GreenSchwarz superstring which can be constructed in any spacetime dimension. Their action is [5]

$$
\begin{aligned}
S= & \int d^{2} \sigma\left[\frac{-g^{a b}}{2 \sqrt{-g}} \Pi_{a}^{\mu} \Pi_{b}^{\mu}-i \epsilon^{a b} \partial_{a} x^{\mu}\left(\bar{\theta} \Gamma^{\mu} \partial_{b} \theta\right)\right. \\
& \left.-i \Lambda^{\mu} \bar{\psi} \Gamma^{\mu} \theta-\frac{1}{\phi} \Lambda^{\mu} \Lambda^{\mu}-\Lambda^{\mu} \epsilon^{a b} \partial_{a} A_{b}^{\mu}\right],
\end{aligned}
$$

where $\Pi_{a}^{\mu}=\partial_{a} x^{\mu}-i \bar{\theta} \Gamma^{\mu} \partial_{a} \theta$.

The first two terms in Eq. (1) are the usual terms in the covariant Green-Schwarz action [6]. The equations of motion from varying the fourth and fifth terms in Eq. (1) imply that $\Lambda^{\mu}$ is a constant null vector. Finally, the equation of motion from varying $\psi$ in the third term eliminates half of the $\theta$ variables. Since half of the $\theta$ variables are eliminated by $\kappa$ symmetry in the standard Green-Schwarz action, the authors conclude that their action describes the correct physi-

\footnotetext{
*E-mail address: nberkovi@power.ift.unesp.br

${ }^{1}$ The first two papers discuss the problem of infinitely reducible first class constraints, while the last three papers discuss the $d$ $=11$ superstring.
}

cal degrees of freedom of the superstring without requiring $\kappa$ symmetry. Furthermore, since their action can be constructed in any dimension, they seemingly avoid the restriction to $D$ $=3,4,6,10$ which follows from requiring $\kappa$ symmetry.

However, there is a problem with the action of Eq. (1). ${ }^{2}$ The problem is that, although $\Lambda^{\mu}$ describes a constant null vector on shell, the direction of this null vector cannot be gauge fixed to point in the direction $\Lambda^{0}=\Lambda^{D-1}=1, \Lambda^{i}=0$, for $i=1$ to $D-2$. In other words, in the light-cone gauge $x^{0}+x^{D-1}=\tau$, the restriction on $\theta$ is $\Lambda^{\mu} \Gamma^{\mu} \theta=0$, rather than the usual $\left(\Gamma^{0}-\Gamma^{D-1}\right) \theta=0$.

So in addition to the usual light-cone degrees of freedom of the Green-Schwarz superstring, one also has the $D-1$ global degrees of freedom given by the constant null modes of $\Lambda^{\mu}$. Unlike the functional integration for the standard Green-Schwarz superstring, functional integration for the Deriglazov-Galajinsky superstring must include an integration over all possible choices of these constant null modes. Therefore, there is no obvious reason why scattering amplitudes using the two different actions should agree.

I would like to thank A. Deriglazov for discussions and CNPq Grant No. 300256/94 for partial financial support.

\footnotetext{
${ }^{2} \mathrm{~A}$ similar problem was earlier noted by Gates [7].
}

[1] A. A. Deriglazov and A. V. Galajinsky, Phys. Rev. D 54, 5195 (1996).

[2] A. A. Deriglazov and A. V. Galajinsky, Phys. Lett. B 381, 105 (1996).

[3] A. A. Deriglazov and A. V. Galajinsky, hep-th/9706152.
[4] A. A. Deriglazov, hep-th/9709025.

[5] A. A. Deriglazov and A. V. Galajinsky, Mod. Phys. Lett. A 112, 2993 (1997).

[6] M. B. Green and J. H. Schwarz, Phys. Lett. 136B, 367 (1984).

[7] S. J. Gates, Jr. (private communication). 